\section{SAT0534 RITUXIMAB FOR REFRACTORY IDIOPATHIC RETROPERITONEAL FIBROSIS: A SINGLE TERTIARY CENTER EXPERIENCE}

M. Oztas ${ }^{1}$, E. Cerme ${ }^{1}$, S. Ugurlu ${ }^{1} .{ }^{1}$ Istanbul University-Cerrahpasa, Department of Medicine, Division of Rheumatology, Istanbul, Turkey

Background: Idiopathic retroperitoneal fibrosis (RPF) is a progressive disorder of the retroperitoneum which is often idiopathic. Although prednisolone is the mainstay approach to treating RPF, the remission rates range between $75 \%$ to $95 \%$ (1-2). Objectives: Here, we report the outcomes and steroid-sparing effect of Rituximab (Rtx) therapy in 14 patients with RPF.

Methods: This retrospective study was conducted at a tertiary rheumatology center. Patients were diagnosed with RPF and had at least a course of $0.5-1 \mathrm{mg} /$ $\mathrm{kg}$ prednisolone treatment previously. These patients were switched to Rtx due to inadequate response or side effects while on prednisone, tamoxifen, azathioprine or cyclophosphamide therapy. Patients were treated with Rtx in order to be included in this study. Involvement and activation of RPF was shown via PET-CT either before or at least 6 months after the therapy. Daily prednisolone dose was noted before rituximab initiation and 6 months after the therapy. All of the patients reported, except two, were followed for at least 6 months after the Rtx treatment. The final disease status of the three patients were not included in the study. Results: Fourteen patients (7F) received at least 2 cycles ( $1 \mathrm{gr}$ for each) of Rtx. The age of diagnosis was $54.3 \pm 11.0$ years, follow-up duration was $46.0 \pm 37.2$ months. The previous treatments, number of the cycles of Rtx and final disease status were shown in the Table. The Control PET-CT revealed metabolic and radiologic remission in 3 patients. In 6 patients, the disease remained stable. In 2 patients there was disease progression hence they were treated with the second course of Rtx. One of the two patients had the progression two years after the first cycle but then, was lost to follow-up. The mean prednisolone dose decreased from $15.5 \pm 12.4 \mathrm{mg}$ to $2.2 \pm$ $2.2 \mathrm{mg} / \mathrm{day}$ after 6 months of Rtx initiation. Final prednisolone dose was $2.6 \pm 5.5 \mathrm{mg} /$ day (Figure). Rtx treatment was ceased in 6 patients with sustained remission.

Conclusion: The present study shows that Rtx could be a therapeutic option after gluocorticoid or DMARD failure. The steroid sparing effect of Rtx is essential and further prospective studies are needed to assess the Rtx efficacy more objectively in RPF treatment.

Table. Characteristics and final disease status of the patients

\begin{tabular}{ccccc}
\hline Number & $\begin{array}{c}\text { Age of Rituximab } \\
\text { Initiation }\end{array}$ & Sex Previous Treatments & $\begin{array}{c}\text { Number of } \\
\text { Rituximab Cycle(s) }\end{array}$ & Final Pet-CT \\
\hline 1 & 49 & M Pred, Mtx & 1 & Stable disease \\
2 & 54 & M Pred,Mtx & 1 & Stable disease \\
3 & 46 & F Pred,Aza,Tmx,Mmf & 2 & Progression \\
4 & 40 & M Pred,Aza,Mtx & 4 & Remission \\
5 & 63 & F Pred,Tmx & 10 & Stable disease \\
6 & 47 & F Pred,Mtx & 2 & Stable disease \\
7 & 30 & F Pred & 1 & Stable disease \\
8 & 52 & M Pred,Aza & 2 & Progression \\
9 & 54 & M Pred & 1 & N/A \\
10 & 59 & M Pred,Aza,Mtx & 1 & N/A \\
11 & 30 & F Pred, Mtx & 6 & Remission \\
12 & 40 & F Pred,Aza,Tmx;Cyc & 3 & Stable disease \\
13 & 50 & M Pred & 2 & N/A \\
14 & 45 & F Pred,Aza & 3 & Remission \\
\hline Pred:Prednisolone, & & & & Aza:Azathioprine, \\
\hline
\end{tabular}

Tmx:Tamoxifen,Mtx:Methotrexate,Cyc:cyclophosphamide

Figure: Corticosteroids sparing effects of Rituximab

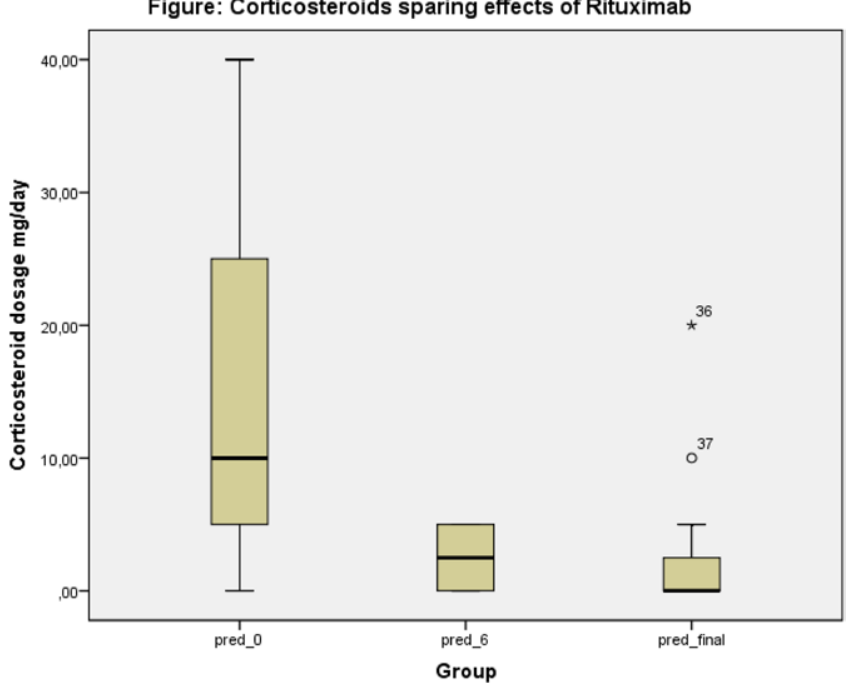

\section{References:}

[1] Vaglio A, Palmisano A, Alberici F, Maggiore U, Ferretti S, Cobelli R, Ferrozzi F, Corradi D, Salvarani C, Buzio C: Prednisone versus ta- moxifen in patients with idiopathic retroperitoneal fibrosis: an open-label randomised controlled trial. Lancet 378: 338-346, 2011

[2] van Bommel EF, Siemes C, Hak LE, van der Veer SJ, Hendriksz TR: Longterm renal and patient outcome in idiopathic retroperito- neal fibrosis treated with prednisone. Am J Kidney Dis 49: 615-625, 2007

Disclosure of Interests: None declared

DOI: 10.1136/annrheumdis-2020-eular.6419

\section{SAT0535 \\ CLINICAL COURSE IN PATIENTS WITH INTERSTITIAL PNEUMONIA WITH AUTOIMMUNE FEATURES (IPAF) IN A MULTIDISCIPLINARY CONSULTATION.}

I. Perez $^{1}$, C. Vadillo ${ }^{1}$, M. A. Nieto ${ }^{2}$, D. Freites ${ }^{1,3}$, Z. Rosales ${ }^{1}$, A. Mucientes ${ }^{3}$, L. León ${ }^{3}$, J. Font ${ }^{1}$, J. A. Jover ${ }^{1}$, L. Abasolo ${ }^{3} .{ }^{1}$ Hospital Clinico San Carlos, Rheumatology, Madrid, Spain; ${ }^{2}$ Hospital Clinico San Carlos, Pneumology, Madrid, Spain; ${ }^{3}$ Fundacion para la Investigación Biomedica - HCSC, Rheumatology, Madrid, Spain

Background: A proportion of patients with Interstitial Lung Disease (ILD) show autoimmune characteristics but do not completely meet the classification criteria for a definitive connective tissue disease. In order to unify the nomenclature and criteria to define this condition, the classification of patients with Interstitial Pneumonia with Autoimmune Features (IPAF) has recently been adopted (Fisher, et al).

Objectives: To describe the sociodemographic, clinical, functional characteristics and therapeutic management of IPAF patients in clinical practice and to evaluate the incidence rate of functional respiratory impairment over time.

Methods: A longitudinal observational study was performed. Patients with IPAF classification criteria (Fischer et al) were included from the time of ILD diagnosis (Feb 2017 to Sept 2018) and followed until loss of follow-up or end of the study (Oct 2019), in a multidisciplinary team, carried by a pneumologist and a rheumatologist in a Tertiary Hospital in Madrid. Main outcome: relative functional respiratory impairment: defined as decline in percent predicted forced vital capacity (FVC\%) of $\geq 5 \%$ compared to the previous visit. Respiratory function was measured at baseline and every 6 months. Covariates: a) sociodemographic, b) clinical, c) radiological pattern (non-specific interstitial pneumonia [NSIP]; usual interstitial pneumonia [UIP], others); d) FVC\%, DLCO\%; e) laboratory tests; f) therapy used (glucorticosteroids disease modifying antirheumatic drugs (DMARDs) and Biologic Agents). Statistical analysis: description of the sociodemographic, clinical, radiological, functional and treatment characteristics of the patients. Survival techniques were used to estimate the incidence rate $(\mathrm{IR})$ of relative functional respiratory impairment, expressed per 100 patient-semester with their respective confidence interval $[95 \% \mathrm{Cl}]$.

Results: 17 patients were included with a mean follow-up of $3 \pm 1.5$ years, $70.6 \%$ were women with a mean age of $65 \pm 10$ years. The most frequent IPAF classification criteria were: a) clinical: arthritis (50\%), Raynaud's phenomenon (33\%) and mechanical hands (17\%); b) serological: $65 \%$ had ANA $\geq 1 / 360 ; 31 \%$ FR> 40; 30\% Anti-Ro positive; c) morphologic: $59 \%$ presented NSIP pattern and $29.4 \%$ was UIP. The baseline median FVC\% and DLCO\% were 89 [83-107.7] and 63 [50-79.8] respectively. During the study period, $94 \%$ received treatment: $87.5 \%$ glucorticosteroids, $68.5 \%$ mycophenolate, $56 \%$ azathioprine, $18.7 \%$ cyclophosphamide iv and $33 \%$ antifibrotics. During the follow-up (104.6 patient - semester), 15 patients presented relative functional respiratory impairment, with an IR of 23.8 [16.1-35.3]. After 14 months from IPAF diagnosis $50 \%$ of the patients had relative functional respiratory impairment. At the end of the follow-up, $50 \%$ showed a worsening of the DLCO $\%$.

Conclusion: IPAF patients are mostly women in their sixties. The most frequent clinical criteria are arthritis and Raynaud's phenomenon and the serological were FR and ANAs. The most frequent radiological pattern was NSIP. The therapeutic management is mainly with glucorticosteroids, mycophenolate and azathioprine. At the beginning, patients have a slightly diminished lung function. These patients have significant functional impairment over time that will impact in their prognosis. Longitudinal and multicenter studies are necessary to advance in the knowledge and management of these patients.

References:

[1] Fischer A, Antoniou KM, Brown KK, Cadranel J, Corte TJ, du Bois RM, et al. An official European Respiratory Society/American Thoracic Society research statement: interstitial pneumonia with autoimmune features. Eu Respir J [Internet]. 2015 Oct;46(4):976-87. Available from: http://www.ncbi. nlm.nih.gov/pubmed/26160873

Disclosure of Interests: None declared

DOI: 10.1136/annrheumdis-2020-eular.4607

\section{SAT0536 IMMUNE CHECKPOINT INHIBITOR THERAPY IN PATIENTS WITH PREEXISTING SARCOIDOSIS}

X. Pundole ${ }^{1}$, O. Lambotte ${ }^{2}$, M. Ramos-Casals ${ }^{3}$, M. Suarez-Almazor ${ }^{1}{ }^{1}$ The University of Texas MD Anderson Cancer Center, Houston, United States of 